\title{
Epicrania fugax combining forward and backward paroxysms in the same patient: the first four cases
}

This article was published in the following Dove Press journal:

Journal of Pain Research

23 June 2017

Number of times this article has been viewed

\author{
Johanna Barón-Sánchez' \\ Álvaro Gutiérrez-Viedma ${ }^{2}$ \\ Marina Ruiz-Piñero' \\ Alicia Pérez-Pérez ${ }^{2}$ \\ Ángel Luis Guerrero',3 \\ María L Cuadrado 2,4 \\ 'Headache Unit, Department \\ of Neurology, Hospital Clínico \\ Universitario de Valladolid, Valladolid, \\ Spain; ${ }^{2}$ Department of Neurology, \\ Hospital Clínico San Carlos, Madrid, \\ Spain; ${ }^{3}$ Department of Medicine, \\ School of Medicine, Universidad \\ de Valladolid, Valladolid, Spain; \\ ${ }^{4}$ Department of Medicine, School of \\ Medicine, Universidad Complutense \\ de Madrid, Madrid, Spain
}

Background: The first description of epicrania fugax (EF) reported brief painful paroxysms that start in posterior regions of the scalp and move forward to reach the ipsilateral forehead, eye, or nose. A backward variation, wherein pain stems from frontal areas and radiates to the posterior scalp, has also been acknowledged. We report four patients with features reminiscent of EF and the coexistence of forward and backward pain paroxysms.

Methods: We considered all patients attending the headache outpatient office at two tertiary hospitals from March 2008 to March 2016. We enrolled four patients with paroxysms fulfilling criteria for EF and a combination of forward and backward radiations.

Results: In all cases, pain paroxysms moved both in forward and backward directions with either a zigzag $(n=2)$ or linear $(n=2)$ trajectory. Three patients presented two stemming points, in the occipital scalp and forehead $(n=2)$ or in the parietal area and eye $(n=1)$, whereas the fourth patient only had a stemming point located in the parietal region. Pain quality was mainly stabbing, and its intensity was moderate $(n=1)$ or severe $(n=3)$. The duration of the paroxysms was highly variable (3-30 seconds), and two patients reported autonomic symptoms.

Conclusion: The clinical picture presented by our patients does not fit with other types of known headache or neuralgia syndromes; we propose it corresponds to a bidirectional variant of EF.

Keywords: epicrania, epicranial neuralgias, backward radiation, forward radiation

\section{Introduction}

Epicrania fugax $(\mathrm{EF})$ is a recently described headache syndrome. ${ }^{1}$ It has been classified as a primary headache in the research Appendix of the 3rd edition of the International Classification of Headache Disorders (ICHD-III, beta version). ${ }^{2}$ EF is characterized by the occurrence of brief pain paroxysms moving along a hemicranium between two distant points through the territories of different nerves. The intensity of pain can be moderate or severe, and its quality has been mainly described as electric or stabbing. In some cases the pain is accompanied by autonomic signs such as conjunctival injection, lacrimation, or rhinorrhea. Between the paroxysms, a slightly painful sensation may persist at the stemming point. Pain frequency is highly variable. Most pain attacks are spontaneous, but some of them can be triggered by tactile stimulation ${ }^{1,3}$ or with cranio- or orofacial movements. ${ }^{4}$

Initial descriptions depicted a unilateral pain spreading from posterior cranial areas to the ipsilateral anterior scalp, following a linear or zigzag trajectory - that is the classical forward EF. ${ }^{1,5}$ Later on, a backward EF - with pain radiating from frontal areas toward the posterior scalp - was also described, with no demographic or clinical differences between both variants. ${ }^{6-8}$ The phenotype of EF has become even broader
Correspondence: Ángel Luis Guerrero Headache Unit, Department of Neurology, Hospital Clínico Universitario de Valladolid, Avda Ramón y Cajal 3, 47005 Valladolid, Spain

Tel +34983420000

Email gueneurol@gmail.com 
during the last few years. For instance, it has been reported that pain paroxysms may occur at shifting sides, ${ }^{1,7}$ along the mid-sagittal plane, ${ }^{9}$ with coronal radiation ${ }^{10}$ or even in a multidirectional fashion. ${ }^{11} \mathrm{~A}$ facial variant of $\mathrm{EF}^{4}$ has also been proposed.

We aim to report four new patients who fulfill EF diagnostic criteria, and present both forward and backward pain paroxysms.

\section{Methods}

We retrospectively considered all patients attending the headache outpatient office at two tertiary hospitals (Hospital Clínico Universitario, Valladolid, and Hospital Clínico San Carlos, Madrid) from March 2008 to March 2016. We selected four patients in whom pain paroxysms moved with both forward and backward radiation. Patients were referred either from primary physicians or general neurology offices; we selected cases among those describing brief and dynamic pain paroxysms. In each case, we considered both demographic and clinical characteristics, including location of the stemming and ending points, pain trajectory, pain quality, pain intensity, accompaniments, and triggers. Neurological examination was unremarkable in all patients, and magnetic resonance imaging (MRI) and laboratory tests were performed in all cases to exclude underlying diseases. Written, informed consent to publish the case details was provided by the patients in this report and has also been obtained from the authors (ALG and MLC). This study was approved by the Research Committee of Hospital Clínico Universitario de Valladolid.

\section{Results}

\section{Patient I}

A 71-year-old woman with a history of menstrual-related migraine was assessed due to a 1-year history of a new type of headache. Pain paroxysms stemmed at the right parietal scalp and spread in a zigzag trajectory, both forwards and backwards, to reach the ipsilateral eye after 1 second and the suboccipital region in 3 seconds (Figure 1A). Pain quality was described as electric, and its intensity was rated as 8 out of 10 on a visual analog scale (VAS; 0 : no pain, 10: the worst imaginable pain). The headache was consistently associated with ipsilateral ptosis, lacrimation, and conjunctival injection. These pain paroxysms appeared from one to countless times throughout the day, with an average frequency of four times per day. A mild interictal pain in a non-circumscribed area persisted over the stemming point. These paroxysms could not only be triggered by touching the parietal scalp but also occurred spontaneously. Physical examination showed hyper-

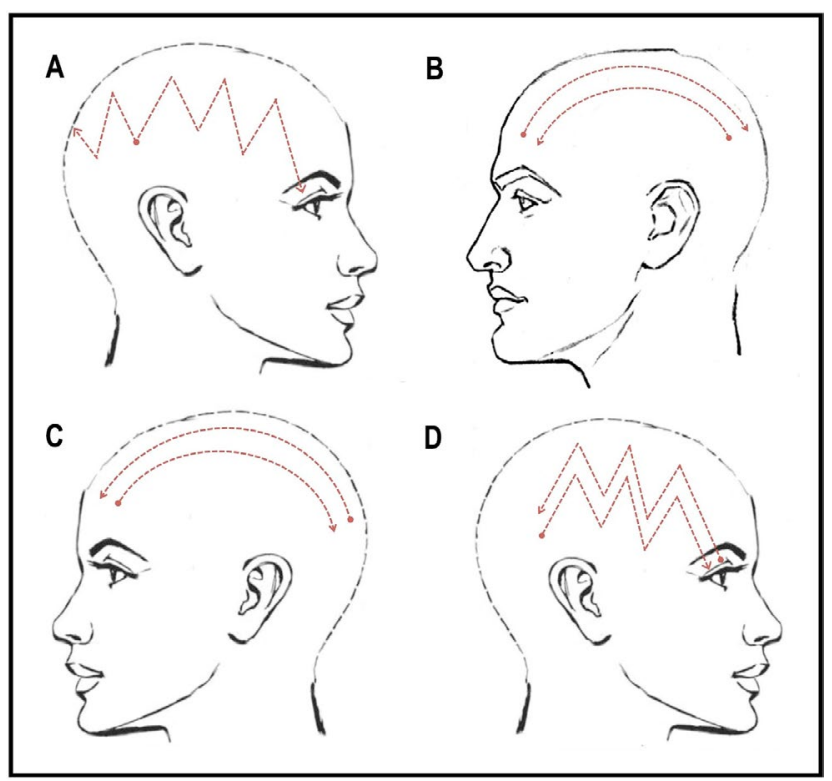

Figure I Trajectory of pain paroxysms in our four patients. (A) Parietal to occipital area and parietal area to the eye (right side). (B) Occipital area to the forehead (right and left sides). (C) Occipital area to the forehead (left side). (D) Parietal area to the eye (right side).

sensitivity upon palpation of the right parietal scalp. Gabapentin (300 mg bid) was prescribed with marked improvement.

\section{Patient 2}

A 42-year-old man with no relevant medical history came to our office due to a 5-year history of pain paroxysms along both hemicrania. Approximately $70 \%$ of these paroxysms radiated in a lineal trajectory from the occipital scalp to the ipsilateral forehead, whereas 30\% moved from the forehead to the ipsilateral occipital region. Most of the paroxysms $(80 \%)$ were located on the right hemicranium, but a minority (20\%) occurred on the left side (Figure 1B). Pain quality was described as stabbing, and pain intensity was rated as 8 out of 10 on a VAS. The attacks lasted 5 seconds and were infrequent (2-3 times a week). There were no associated symptoms. The patient did not report other interictal tenderness or pain triggers. Lamotrigine $100 \mathrm{mg}$ bid was prescribed, leading to substantial pain relief.

\section{Patient 3}

A 21-year-old woman with prior history of migraine with visual aura started suffering a new type of headache 1 year before assessment. She complained of two paroxysms per week comprising a stabbing pain moving with a linear trajectory between the left occipital region and the ipsilateral forehead lasting for 20 seconds. Approximately $90 \%$ of the paroxysms radiated backwards, but $10 \%$ had forward radiation (Figure 1C). Pain intensity was rated as 6 out of 10 on a VAS. All of these attacks were associated with ipsilateral lacrimation. The patient did not report any precipitating 
factors and described no pain during interictal periods. Lamotrigine $100 \mathrm{mg}$ bid was recommended, but adherence to therapy was incomplete.

\section{Patient 4}

A 57-year-old woman with no prior headaches presented with an 8-month history of paroxysmal pain. In most instances $(90 \%)$, the pain started over the right parietal scalp and moved in a zigzag manner to the ipsilateral eye. Yet, $10 \%$ of the episodes had backward radiation from the right eye to the ipsilateral occipital region (Figure 1D). The pain was described as stabbing and extremely severe (10 out of 10 ). Attacks lasted approximately 30 seconds, and there were no accompanying symptoms. Paroxysms were more frequent at night and occurred three times a week. The patient reported neither interictal pain nor tenderness. Treatment with amitriptyline (10 mg q.d.) was only partially effective.

\section{Discussion}

We describe four new patients with characteristics considered distinctive of EF. Each of them presented with bidirectional paroxysms of pain, with the pain moving both forwards and backwards. Forward and backward paroxysms could occur at the same time (Patient 1) or in different attacks (Patients 2-4). Pain quality and intensity, as well as the radiation with a linear or zigzag trajectory, did not depend on the direction of the pain. The duration of the attacks was variable, between 3 and 30 seconds. In two of the cases (Patients 1 and 3), the pain was associated with autonomic signs. Between attacks, one patient (Patient 1) had mild pain and tenderness at the stemming area.

The clinical picture of EF has been described as a combination of static and dynamic components. Pain paroxysms run between the posterior scalp (C2) and the frontal or periorbital area (V1), either in a forward or a backward direction. With regard to the static component, the stemming area can be slightly painful between the paroxysms (12). In the facial variant of EF, pain starts in the face (dermatomes V2 or V3). ${ }^{4}$

The etiology of EF remains unknown, although clinical characteristics such as stabbing character of the pain and the presence of a stemming area suggest an epicranial source. ${ }^{12}$ It has been proposed that the pain may start at the terminal branches of the pericranial nerves and that pain spreads due to different mechanisms, such as aberrant ephaptic transmission connecting different nerve fibers, involvement of transdiploic fibers, ${ }^{1}$ or recruitment of central mechanisms. ${ }^{12}$

The static component of EF has been related to the area in which the pain initiates. This baseline pain has been more frequently described among patients with forward radiation, than in those with backward variant. We have also observed in some cases an interictal pain in the area in which pain paroxysms end (data not published). Similarly as a nummular headache may be located in different scalp areas in the same patient, ${ }^{13}$ peripheral generators of EF may be located in anterior or posterior regions, ${ }^{12}$ as happened in three of our cases. In our opinion, therefore, it is not surprising to have the appearance of forward and backward radiations in the same patient as described in this series.

\section{Conclusion}

The patients in our series fulfilled diagnostic criteria of EF, with a paroxysmal pain that manifested in two directions (forward and backward) in the same patient at different times or simultaneously. This description expands the clinical spectrum of EF and further clarifies the differential diagnosis between EF and pericranial neuralgias.

\section{Acknowledgments}

The abstract of this paper was presented at the 2 nd Congress of the European Academy of Neurology as a poster presentation with interim findings. The poster's abstract was then published in the European Journal of Neurology. vol 23, June 2016.

\section{Disclosure}

The authors report no conflicts of interest in this work.

\section{References}

1. Pareja JA, Cuadrado ML, Fernández-de-las-Peñas C, et al. Epicrania fugax: an ultrabrief paroxysmal epicranial pain. Cephalalgia. 2008;28(3):257-263.

2. Headache Classification Committee of the International Headache Society (IHS). The International Classification of Headache Disorders, 3rd edition (beta version). Cephalalgia. 2013;33(9):629-808.

3. Mulero P, Guerrero AL, Herrero-Velázquez S, et al. Epicrania fugax with backward radiation: clinical characteristics of nine new cases. $J$ Headache Pain. 2011;12(5):535-539.

4. Cuadrado ML, Aledo-Serrano Á, Jiménez-Almonacid J, de Lera M, Guerrero ÁL. Facial pain radiating upwards: could the pain of epicrania fugax start in the lower face? Headache. 2015;55(5):690-695.

5. Guerrero AL, Cuadrado ML, Porta-Etessam J, et al. Epicrania fugax: ten new cases and therapeutic results. Headache. 2010;50(3):451-458.

6. Herrero-Velázquez S, Guerrero-Peral ÁL, Mulero P, et al. picrania fugax: Características clínicas de una serie de 18 pacientes. [Epicrania fugax: the clinical characteristics of a series of 18 patients]. Rev Neurol. 2011;53(9):531-537. Spanish [with English abstract].

7. Cuadrado ML, Ordás CM, Sánchez-Lizcano M, et al. Epicrania fugax: 19 cases of an emerging headache. Headache. 2013;53(5):764-774.

8. Cuadrado ML, Gómez-Vicente L, Porta-Etessam J, Marcos-de-Vega MA, Parejo-Carbonell B, Matias-Guiu J. Paroxysmal head pain with backward radiation: will epicrania fugax go in the opposite direction? J Headache Pain. 2010;11(1):75-78.

9. de la Cruz C, Herrero-Velázquez S, Ruiz M, et al. Epicrania fugax with sagittal trajectory of the pain. Pain Med. 2015;16(6):1238-1239. 
10. Casas-Limón J, Cuadrado ML, Ruiz M, et al. Pain paroxysms with coronal radiation: case series and proposal of a new variant of epicrania fugax. Headache. 2016;56(6):1040-1044.

11. Cuadrado ML, Aledo-Serrano A, Di Capua D, Pareja JA. A multidirectional epicrania fugax. Cephalalgia. 2015;35(9):835-836.
12. Cuadrado ML, Guerrero AL, Pareja JA. Epicrania fugax. Curr Pain Headache Rep. 2016;20(4):21.

13. Pareja JA, Caminero AB, Serra J, Barriga FJ, Barón M, Dobato JL. Numular headache: a coin-shaped cephalgia. Neurology. 2002;58(11): $1678-1679$.

\section{Publish your work in this journal}

The Journal of Pain Research is an international, peer reviewed, open access, online journal that welcomes laboratory and clinical findings in the fields of pain research and the prevention and management of pain. Original research, reviews, symposium reports, hypothesis formation and commentaries are all considered for publication.
Dovepress

The manuscript management system is completely online and includes a very quick and fair peer-review system, which is all easy to use. Visit http://www.dovepress.com/testimonials.php to read real quotes from published authors. 